\title{
Spin correlations in the electron-doped high-transition-temperature superconductor $\mathrm{Nd}_{2-x} \mathrm{Ce}_{x} \mathrm{CuO}_{4 \pm \delta}$
}

\author{
E.M. Motoyama, ${ }^{1}$ G. Yu,${ }^{1}$ I.M. Vishik, ${ }^{1}$ O.P. Vajk, ${ }^{2}$ P.K. Mang,${ }^{3}$ and M. Greven ${ }^{3,4, \text { 冈 }}$ \\ ${ }^{1}$ Department of Physics, Stanford University, Stanford, California 94305, USA \\ ${ }^{2}$ NIST Center for Neutron Research, National Institute of Standards and Technology, Gaithersburg, Maryland 20899, USA \\ ${ }^{3}$ Department of Applied Physics, Stanford University, Stanford, California 94305, USA \\ ${ }^{4}$ Stanford Synchrotron Radiation Laboratory, Stanford, California 94309, USA
}

High-transition-temperature (high- $T_{\mathrm{c}}$ ) superconductivity develops near antiferromagnetic phases, and it is possible that magnetic excitations contribute to the superconducting pairing mechanism. To assess the role of antiferromagnetism, it is essential to understand the doping and temperature dependence of the twodimensional antiferromagnetic spin correlations. The phase diagram is asymmetric with respect to electron and hole doping, and for the comparatively less-studied electron-doped materials, the antiferromagnetic phase extends much further with doping [1, 2] and appears to overlap with the superconducting phase. The archetypical electron-doped compound $\mathrm{Nd}_{2-x} \mathrm{Ce}_{x} \mathrm{CuO}_{4 \pm \delta}$ (NCCO) shows bulk superconductivity above $x$ $\approx 0.13[3,4]$, while evidence for antiferromagnetic order has been found up to $x \approx 0.17[2,5,[6]$. Here we report inelastic magnetic neutron-scattering measurements that point to the distinct possibility that genuine long-range antiferromagnetism and superconductivity do not coexist. The data reveal a magnetic quantum critical point where superconductivity first appears, consistent with an exotic quantum phase transition between the two phases [7]. We also demonstrate that the pseudogap phenomenon in the electron-doped materials, which is associated with pronounced charge anomalies $[8,9,10,11]$, arises from a buildup of spin correlations, in agreement with recent theoretical proposals [12, 13].

In their as-grown state, the electron-doped materials exhibit antiferromagnetic (AF) order throughout the accessible doping range, and an oxygen reduction treatment is required to induce superconductivity [3]. Previous inelastic neutron scattering experiments on as-grown, nonsuperconducting (non-SC) NCCO demonstrated [6] that the two-dimensional (2D) spin correlation length $\xi$ observed above the Néel temperature is exponentially dependent on inverse temperature:

$$
\xi(x, T)=A(x) \exp \left(2 \pi \rho_{s}(x) / T\right)
$$

for cerium concentrations ranging from zero up to the solubility limit of $x \approx 0.18$. This behaviour indicates the existence of an underlying ground state with long-range 2D AF order. Owing to weak spin-space anisotropies and three-dimensional couplings, NCCO exhibits threedimensional AF order at a non-zero Néel temperature, as observed in the elastic scattering channel [2, 6]. The spin stiffness $\rho_{s}(x)$ decreases monotonically with increasing electron concentration, with $\rho_{s}(0.18) / \rho_{s}(0) \approx 25 \%[6]$, and the doping dependence of $\rho_{s}(x)$ and of the amplitude $A(x)$ is remarkably close to that for the randomly-diluted spin-one-half square-lattice Heisenberg antiferromagnet $\mathrm{La}_{2} \mathrm{Cu}_{1-z}(\mathrm{Zn}, \mathrm{Mg})_{z} \mathrm{O}_{4}$ [14].

Magnetic inelastic neutron-scattering experiments in the SC phase have become possible only in recent years [15, 16]. We have carried out two-axis measurements of the spin correlations in eight oxygen-reduced NCCO crystals in the cerium concentration range $0.038 \leq x \leq 0.154$ (Fig. 11). The data are fit to a $2 \mathrm{D}$ lorentzian, $S\left(q_{2 \mathrm{D}}\right)=$ $S(0) /\left(1+q_{2 \mathrm{D}}^{2} \xi^{2}\right)$, convoluted with the calculated instrumental resolution, where $q_{2 \mathrm{D}}$ is the distance in momentum space from the 2D AF zone center (Fig. 2). The non-SC samples $(x \leq 0.129)$ follow equation (1) , consistent with bulk AF order in the ground state (Fig. 3). Although this behaviour is qualitatively the same as that for as-grown NCCO [6], the spin stiffness decreases much more rapidly with doping. The data for the SC sample with $x=0.134$ are fit to equation (11) with a small value of the spin stiffness $\rho_{s}$, but are equally well described by the simple power law $\xi \propto 1 / T^{\nu_{T}}$ with exponent $\nu_{T}=1.0(5)$. The power-law behaviour, indicated by the dashed curve in Fig. 3. would imply that $\rho_{s}$ is already zero and that the system is quantum critical at this cerium concentration. Fig. 植 demonstrates that $\rho_{s}$ approaches zero at $x_{\mathrm{AF}}=0.134(4)$ in an approximately linear fashion. In a fundamental departure from the above behaviour, we find that in the SC samples with $x \geq 0.145, \xi$ remains finite down to the lowest temperatures. The low-temperature correlation length $\xi_{0}$ for these samples increases as $x_{\mathrm{AF}}$ is approached from above (Fig. $4 \mathrm{k}$ ).

Previous elastic neutron measurements [2, 4, 6] indicated that oxygen-reduced NCCO exhibits AF order up to $x \approx 0.17$. Such measurements in our crystals indeed reveal Néel order. However, our inelastic results demonstrate that, contrary to previous belief [4, 11, 17], the ground state of SC samples exhibits only short-range spin correlations. Moreover, prior inelastic neutron-scattering experiments clearly revealed a SC magnetic gap, despite the presence of $\mathrm{AF}$ Bragg peaks in the elastic response [15, 16]. We conclude that the AF phase boundary in 
fact terminates at $x_{\mathrm{AF}}=0.134(4)$, and that magnetic Bragg peaks observed at higher cerium concentrations originate from regions of the samples which were not fully oxygen-annealed. While a relatively small volume fraction of such macroscopic remnants of the AF as-grown state can give rise to significant Bragg scattering, our inelastic measurements are fortunately insensitive to their presence. This conclusion is consistent with the observation that the Néel transition is very broad in SC samples (Fig. 4b), and also with muon spin-resonance results [4], which show a significant decrease of the AF volume fraction near $x=0.14$. We note that the spurious elastic signal from the remnant AF regions should not be confused with the spurious elastic signal in a magnetic

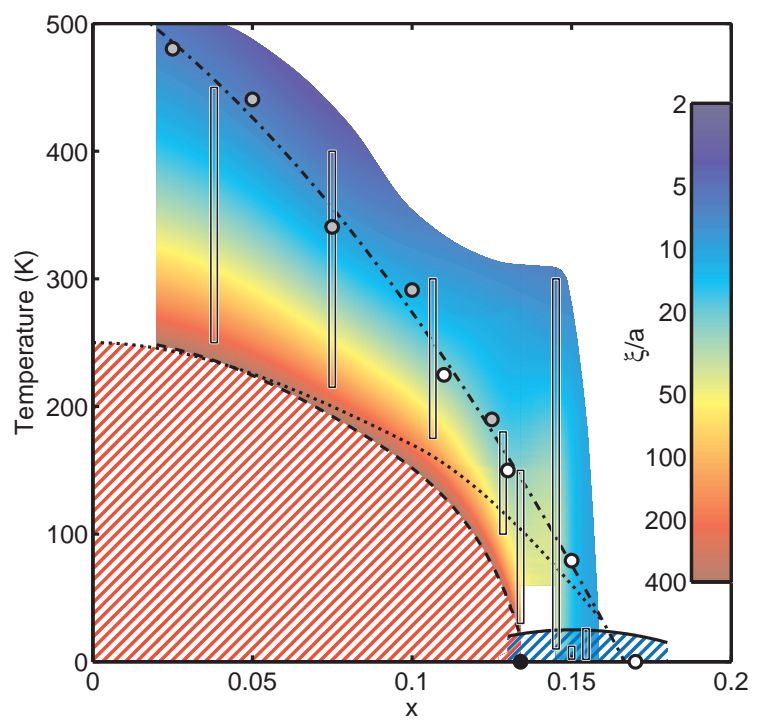

FIG. 1: The temperature-doping phase diagram for oxygen-reduced NCCO. The red and blue hashed areas indicate long-range AF order and superconductivity, respectively. The black circle at zero temperature indicates the approximate location of a magnetic quantum phase transition. The instantaneous $2 \mathrm{D}$ spin-correlation length $\xi(x, T)$ in the $\mathrm{CuO}_{2}$ sheets was measured at the doping levels and over the temperature ranges indicated by the vertical bars. The colour scale shows $\xi$, in units of the planar lattice constant $a$, interpolated and extrapolated from the measured values. The Néel temperature $T_{\mathrm{N}}$ is shown as the dotted curve, while the dashed curve is the extrapolated contour of $\xi / a=400$. The measurement of $T_{\mathrm{N}}$ is contaminated by remnants of the as-grown state of $\mathrm{NCCO}$, so that the true AF phase extends only to $x_{\mathrm{AF}} \approx 0.13$, close to where superconductivity first appears. This is established from the fact that $\xi$ diverges exponentially upon cooling for non-superconducting compositions at lower electron concentrations, while it remains finite in superconducting samples. The small remaining overlap indicated in the figure may be caused by cerium and oxygen inhomogeneities. The grey and white circles indicate optical conductivity measurements of of the pseudogap temperature $T^{*}$ on NCCO crystals [9] and $\operatorname{Pr}_{2-x} \mathrm{Ce}_{x} \mathrm{CuO}_{4 \pm \delta}$ thin films [1] , respectively. The dot-dashed curve is a guide to the eye. field [18] due to the paramagnetic decomposition product $(\mathrm{Nd}, \mathrm{Ce})_{2} \mathrm{O}_{3}$.

For as-grown NCCO [6] and for $\mathrm{La}_{2} \mathrm{Cu}_{1-z}(\mathrm{Zn}, \mathrm{Mg})_{z} \mathrm{O}_{4}$ 14] it was found that, to a very good approximation, the

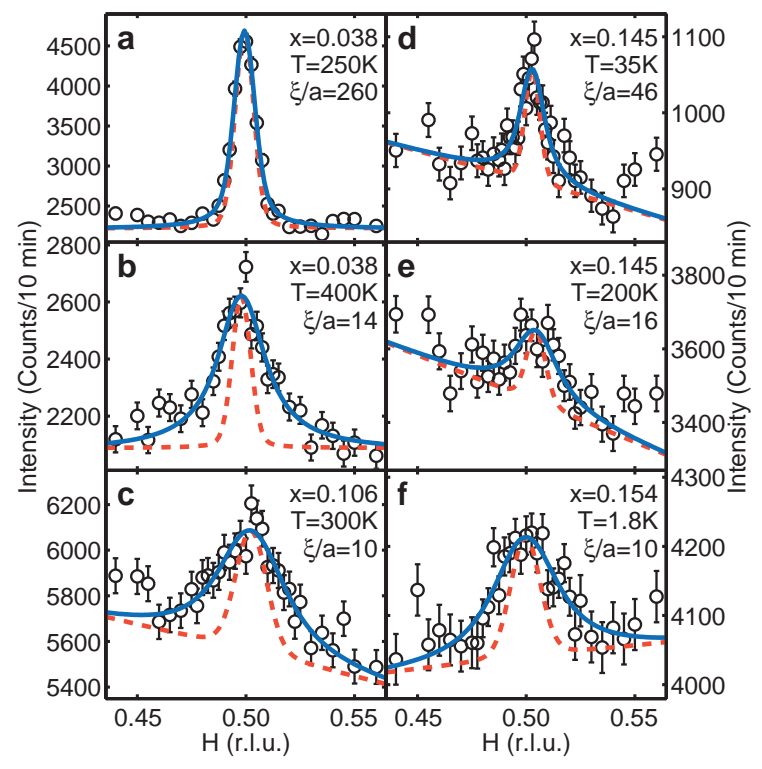

FIG. 2: Representative two-axis scans used to measure the spin correlation length. The scans are along $(h, h)$ about the $2 \mathrm{D}$ AF zone centre $(1 / 2,1 / 2)$ and are fitted (solid blue curves) to a $2 \mathrm{D}$ Lorentzian convoluted with the calculated instrumental resolution (dashed red curves). Shown are data at $\mathbf{a}, T=250 \mathrm{~K} \approx T_{\mathrm{N}}$ and $\mathbf{b}, T=400 \mathrm{~K}$ for $x=0.038$; c, $T=300 \mathrm{~K}$ for $x=0.106$; d, $T=35 \mathrm{~K}$ and $\mathbf{e}, T=200 \mathrm{~K}$ for $x=0.145$; and $\mathbf{f}, T=1.8 \mathrm{~K}$ for $x=0.154$. Wavevectors are represented as $(h, k, l)$ in reciprocal lattice units (r.l.u.), where $Q=(2 \pi h / a, 2 \pi k / a, 2 \pi l / c)$ is the momentum transfer, and $a$ and $c$ are the lattice constants of the tetragonal system (space group $I 4 / \mathrm{mmm}$; for $x=0.038$, for example, the room-temperature lattice constants are $a=3.93 \AA$ and $c=12.09 \AA)$. Vertical error bars represent uncertainties $(1 \sigma)$ assuming Poisson statistics. The measurements were performed in two-axis mode on the BT2 and BT9 triple-axis spectrometers at the NIST Center for Neutron Research. The incident neutron energy was $E_{\mathrm{i}}=14.7 \mathrm{meV}$. In previous experiments on $\mathrm{La}_{2} \mathrm{Cu}_{1-z}(\mathrm{Zn}, \mathrm{Mg})_{z} \mathrm{O}_{4}$ [14] and as-grown NCCO [6], this energy proved to be sufficiently large in the temperature region $T_{\mathrm{N}}<T<2 T_{\mathrm{N}}$ to reliably extract the instantaneous structure factor $S(Q)$. The collimations were: a, b, $40^{\prime}-$ $23^{\prime}$-sample- $20^{\prime}$; c, $60^{\prime}-40^{\prime}$-sample- $40^{\prime} ;$ d, $40^{\prime}-47^{\prime}$-sample- $10^{\prime}$; e, $40^{\prime}-47^{\prime}$-sample- $20^{\prime}$; and f, $40^{\prime}-47^{\prime}$-sample- $40^{\prime}$. The NCCO crystals were grown in $4 \mathrm{~atm}$ of oxygen using the travellingsolvent floating-zone technique, and subsequently annealed for $10 \mathrm{~h}$ at $970^{\circ} \mathrm{C}$ in argon, followed by $20 \mathrm{~h}$ at $500^{\circ} \mathrm{C}$ in oxygen. The sample masses range from 1 to $5 \mathrm{~g}$. The oxygen reduction treatment, required for superconductivity to appear, is a non-equilibrium process resulting in unavoidable oxygen inhomogeneities. Cerium concentrations $x$ were determined from inductively coupled plasma (ICP) spectroscopy, with typical variation of $\Delta x \approx 0.005$ along the growth direction. Superconductivity is observed from magnetic susceptibility measurements for $x \geq 0.134$. 
Néel temperature $T_{\mathrm{N}}(x)$ is a contour of constant $2 \mathrm{D}$ correlation length with $\xi / a=200-400$ and 100 , respectively. Following the observations for as-grown NCCO, we plot the extrapolated contour of $\xi / a=400$ as a dashed curve in Fig. 1. This estimate of the underlying bulk Néel temperature coincides with the measured $T_{\mathrm{N}}$ at $x=0.038$, but it lies systematically lower at higher cerium concentrations, approaching $T_{\mathrm{N}}=0$ at $x_{\mathrm{AF}} \approx 0.134$.

The decrease to zero of the spin stiffness at $x_{\mathrm{AF}} \approx$ 0.134 and the finite values of $\xi_{0}$ for $x>x_{\mathrm{AF}}$ indicates a fundamental change in the nature of the magnetic ground state. The contribution of the AF remnants may lead to a slight over-estimate of the spin correlations, and consequently of $\rho_{s}$ and $\xi_{0}$, but we emphasize that the qualitative change in behaviour is a robust result. The NCCO phase diagram resembles those of other unconventional superconductors, such as the heavy-fermion compound $\mathrm{CeRhIn}_{5}$, in which the $\mathrm{AF}$ and $\mathrm{SC}$ phases are believed to be separated by a first-order boundary [19]. Although we cannot rule out a genuine underlying coexistence between $\mathrm{AF}$ and SC order, such coexistence would be confined to a rather narrow doping range. However, the behaviour of $\rho_{s}(x)$, which decreases continuously by more than an order of magnitude with doping, together with the crossover to power-law behaviour of $\xi(x, T)$ near $x=0.134$, suggests another sce-

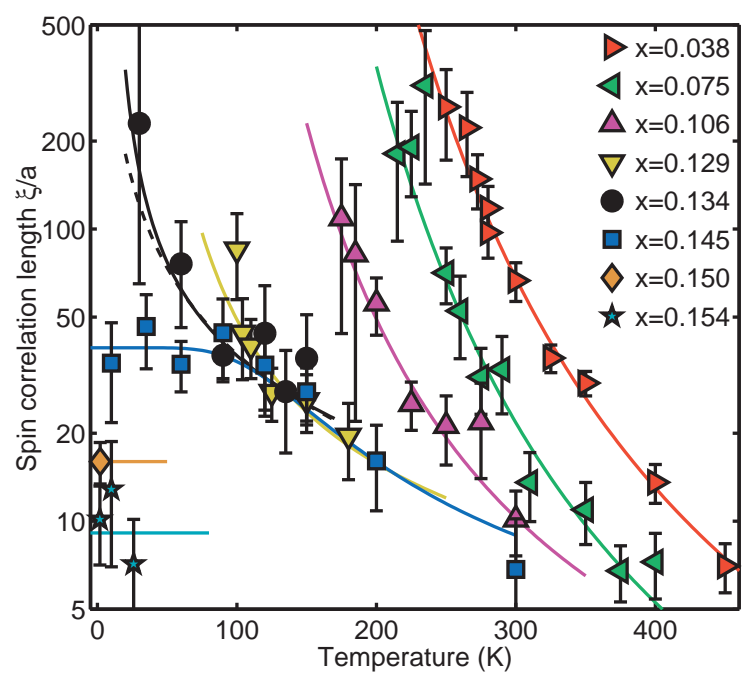

FIG. 3: The temperature dependence of the spin correlation length at various cerium concentrations. Vertical bars represent uncertainties of $1 \sigma$. The data for $x \leq 0.134$ are fit to equation (1). The spin stiffness may already be zero for $x=0.134$, because a fit to a simple power law $\xi \propto 1 / T$ describes the data equally well (dashed curve); power-law behaviour is expected at a quantum critical point. For $x=0.145$ and higher, $\xi$ does not diverge, but instead remains finite at low temperatures, demonstrating the absence of genuine long-range AF order. The curves drawn for these latter data are guides to the eye. Superconductivity is observed for $x \geq 0.134$. nario: a second-order quantum phase transition between the AF and SC phases. This quantum phase transition would be described by a dynamic critical exponent of $z=1 / \nu_{T} \approx 1.0(5)$, which differs from the value $z=2$ predicted for a transition from the antiferromagnet to

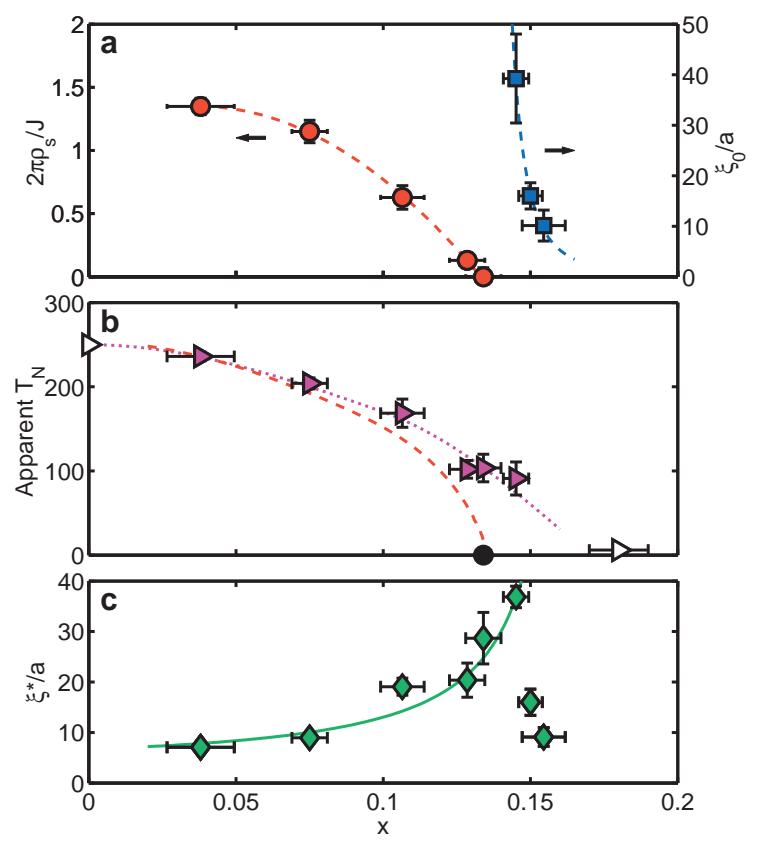

FIG. 4: Spin stiffness, spin correlations at lowtemperature, apparent Néel temperature, and spin correlations along $T^{*}$. a, Doping dependence of the spin stiffness $\rho_{s}$ (plotted as $2 \pi \rho_{s} / J$, where $J=125 \mathrm{meV}$ is the AF superexchange for the undoped Mott insulator $\mathrm{Nd}_{2} \mathrm{CuO}_{4}$ $[2,[6])$ and of the low-temperature spin correlation length $\xi_{0}$. Vertical error bars here represent uncertainties of $1 \sigma$. Horizontal error bars in all panels represent the measured range of cerium concentration in each crystal. Dashed curves are guides to the eye. The spin stiffness decreases smoothly with doping and reaches zero in an approximately linear fashion around $x_{\mathrm{AF}} \approx 0.134$. The ground state for $x<x_{\mathrm{AF}}$ has long-range AF order, whereas long-range order is absent for $x>x_{\mathrm{AF}}$, as seen from the finite values of $\xi_{0}$. The doping dependence of $\xi_{0}$ indicates a divergence as the critical point is approached from the right. b, Apparent Néel temperature $T_{\mathrm{N}}$, as determined from elastic scattering, as a function of doping. The temperature dependence of the measured order parameter (not shown) was modelled using a gaussian distribution of $T_{\mathrm{N}}$, and the vertical bars indicate the full-width at half-maximum (FWHM) of this distribution. Measurements were not performed on all samples; previous data [6] are indicated by open symbols. The dotted and dashed curves are the same as in Fig. 1. c, The spin correlation length $\xi^{*}$ measured at or extrapolated to the pseudogap temperature $T^{*}$. Vertical bars represent uncertainties of $1 \sigma$. Below optimal doping $(x<0.15), \xi^{*}$ is given by the single-particle thermal de Broglie wavelength and increases as $\xi^{*} \propto 1 /\left(x^{*}-x\right)$ (fitted curve). However, this relationship breaks down near optimal doping, where $\xi^{*}$ is found not to exceed the SC coherence length. 
a non-SC paramagnet [12]. If hyperscaling holds, the spin stiffness for a $2 \mathrm{D}$ system is expected to decrease as $\rho_{s} \propto\left(x_{\mathrm{AF}}-x\right)^{\nu_{0} z}$, where $\nu_{0}$ is the exponent describing the divergence of $\xi_{0}$ as $x_{\mathrm{AF}}$ is approached from above. From the approximately linear behaviour of the spin stiffness, we therefore have $\nu_{0} \approx 1$. We cannot independently determine $\nu_{0}$, since we do not have sufficient information for $\xi_{0}(x)$. It is also possible that the system lies above the upper critical dimension, in which case mean-

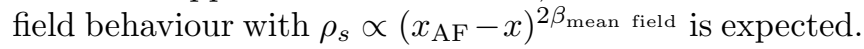
$\beta_{\text {mean field }}=1 / 2$, so this is consistent with the observed behaviour.

Our results for $\xi(x, T)$ also have important consequences for the relationship between AF correlations and the pseudogap physics in the electron-doped copper oxides, which appears to be different from that of the hole-doped materials [9, 12, 13]. The pseudogap (charge anomalies associated with the opening of a partial gap along the Fermi surface) has been discerned in photoemission [8, 10], optical spectroscopy [9, 11], and charge transport [9] experiments on NCCO crystals and $\mathrm{Pr}_{2-x} \mathrm{Ce}_{x} \mathrm{CuO}_{4 \pm \delta}$ films up to $x=0.15$ [8, 11]. First, our finding that the $\mathrm{AF}$ phase terminates at $x_{\mathrm{AF}}=0.134(4)$ refutes statements that a possible quantum phase transition at $x \approx 0.17$ is related to the disappearance of $\mathrm{AF}$ order [11, 17]. Second, we find that the spin-correlation length changes smoothly across the pseudogap temperature $T^{*}$ and, remarkably, up to $x=0.145$ it follows the simple relationship:

$$
\frac{\xi^{*}}{a}=\frac{C}{x_{c}-x}
$$

with fitting parameters $C=0.96(12)$ and $x_{c}=0.171(4)$. One interpretation of the pseudogap is that it signifies a change in the spin scattering of the electrons as the AF correlations exceed the carrier mean free path [9] $(\ell)$ or the single-particle thermal de Broglie wavelength [12] $\left(\xi_{\text {th }}=\hbar v_{F} / \pi k_{B} T\right)$ upon cooling. Boltzmann transport theory, which might be expected to qualitatively hold if $\ell>a$ and $T>T^{*}$, applied to direct-current resistivity data for NCCO yields $\ell\left(T^{*}\right) \approx 2.4 a$ and $\ell\left(T^{*}\right) \approx 25 a$ for $x=0.125$ and $x=0.15$, respectively [9]. This trend is qualitatively consistent with our results up to $x=0.145$ seen in Fig. 45. However, given the approximately linear relationship $T^{*} \propto\left(x^{*}-x\right)$ (Fig. 1), the thermal de Broglie wavelength at $T^{*}$ exhibits the same quantitative doping dependence as does equation (2). Using the value $v_{F}=2.2 \times 10^{7} \mathrm{~cm} / \mathrm{s}$ for the bare Fermi velocity [12], we find that $\xi^{*}=2.6(2) \xi_{\text {th }}$. We conclude that $T^{*}$ is a crossover temperature below which the spin correlations become longer than the thermal de Broglie wavelength, in agreement with theoretical work 12]. The pseudogap phenomenon in the electron-doped copper oxides therefore results from $2 \mathrm{D} \mathrm{AF}$ spin correlations, and does not appear to be a direct precursor to superconductivity. That $\xi^{*} \propto \xi_{\text {th }}$ is obeyed so well suggests that the contributions from the remnant $\mathrm{AF}$ regions to our measured $\xi(x, T)$ are negligible.

Equation (2) would suggest that $\xi^{*}$ diverges at $x_{c} \approx x^{*}$. However, as seen from Fig. 4k, this relation breaks down near optimal doping, presumably owing to the emergence of a new length scale that limits the development of spin correlations. Interestingly, at optimal doping $(x=0.15)$, $\xi^{*} \approx \xi_{0}$ is comparable to the SC coherence length [20] $\xi_{\mathrm{SC}}=58 \AA \approx 15 a$. The spin correlations near optimal doping are still relatively large, consistent with suggestions based on Raman scattering [21] and photoemission 22] that the $d_{x^{2}-y^{2}} \mathrm{SC}$ order parameter is non-monotonic due to AF fluctuations.

The new experimental results for the magnetic phase diagram and pseudogap physics contain important implications for theories of high- $T_{\mathrm{c}}$ superconductivity. By avoiding spurious scattering that significantly contaminates the elastic response, we have established from measurements of the $2 \mathrm{D}$ spin correlations that genuine coexistence of $\mathrm{AF}$ and $\mathrm{SC}$ order is essentially absent in $\mathrm{Nd}_{2-x} \mathrm{Ce}_{x} \mathrm{CuO}_{4 \pm \delta}$. On symmetry grounds, a possible second-order quantum phase transition between these two types of order would seem unlikely and exotic. One scenario is that an underlying first-order transition is rendered second-order owing to microscopic disorder [23]. Such disorder is found in most high- $T_{\mathrm{c}}$ superconductors 24] and, in the present case, it might be the randomness associated with the Nd-Ce substitution. Alternatively, such a phase transition may be an example of 'deconfined' quantum criticality [7], a new paradigm for quantum phase transitions. To further elucidate the nature of the transition from AF to SC order, a detailed complementary study of the superconducting critical properties on small samples with minimal oxygen and cerium inhomogeneities would be desirable.

We thank N. Bontemps, S. Chakravarty, S.A. Kivelson, R.S. Markiewicz and A.-M.S. Tremblay for discussions. The work at Stanford University was supported by grants from the Department of Energy and the National Science Foundation. E.M.M. acknowledges support through the NSF Graduate Fellowship programme.

* greven@stanford.edu

[1] B. Keimer et al., Phys. Rev. B 46, 14034 (1992).

[2] M. Matsuda et al., Phys. Rev. B 45, 12548 (1992).

[3] H. Takagi, S. Uchida and Y. Tokura, Phys. Rev. Lett. 62, 1197 (1989).

[4] T. Uefuji et al., Physica C 357-360, 208 (2001).

[5] T. Uefuji et al., Physica C 378-381, 273 (2002).

[6] P. K. Mang et al., Phys. Rev. Lett. 93, 027002 (2004).

[7] T. Senthil et al., Science 303, 1490 (2004).

[8] N. P. Armitage et al., Phys. Rev. Lett. 88, 257001 (2002).

[9] Y. Onose, Y. Taguchi, K. Ishizaka and Y. Tokura, Phys. Rev. B 69, 024504 (2004).

[10] H. Matsui et al., Phys. Rev. Lett. 94, 047005 (2005). 
[11] A. Zimmers et al., Europhys. Lett. 70, 225 (2005).

[12] B. Kyung, V. Hankevych, A.-M. Daré and A.-M. S. Tremblay, Phys. Rev. Lett. 93, 147004 (2004).

[13] R. S. Markiewicz, Phys. Rev. B 70, 174518 (2004).

[14] O. P. Vajk et al., Science 295, 1691 (2002).

[15] K. Yamada et al., Phys. Rev. Lett. 90, 137004 (2003).

[16] E. M. Motoyama et al., Phys. Rev. Lett. 96, 137002 (2006).

[17] Y. Dagan et al., Phys. Rev. Lett. 94, 057005 (2005).
18] P. K. Mang et al., Phys. Rev. B 70, 094507 (2004).

[19] T. Park et al., Nature 440, 65 (2006).

[20] Y. Wang et al., Science 299, 86 (2003).

[21] G. Blumberg et al., Phys. Rev. Lett. 88, 107002 (2002).

[22] H. Matsui et al., Phys. Rev. Lett. 95, 017003 (2005).

[23] M. Aizenman and J. Wehr, Commun. Math. Phys. 130, 489 (1990)

[24] H. Eisaki et al., Phys. Rev. B 69, 064512 (2004). 